\title{
ON THE MINIMAL NUMBER OF SINGULAR FIBERS IN LEFSCHETZ FIBRATIONS OVER THE TORUS
}

\author{
ANDRÁS I. STIPSICZ AND KI-HEON YUN
}

\begin{abstract}
We show that the minimal number of singular fibers $N(g, 1)$ in a genus- $g$ Lefschetz fibration over the torus is at least 3. As an application, we show that $N(g, 1) \in\{3,4\}$ for $g \geq 5, N(g, 1) \in\{3,4,5\}$ for $g=3,4$ and $N(2,1)=7$.
\end{abstract}

\section{INTRODUCTION}

Due to results of Donaldson and Gompf, the study of symplectic 4-manifolds is closely related to the study of Lefschetz pencils and Lefschetz fibrations. A central open problem in four-manifold theory is the geography problem of symplectic 4manifolds and of Lefschetz fibrations: if $\chi$ and $\sigma$ denote the Euler characteristic and the signature of a 4-manifold, what relations do these integers satisfy and which pairs can be realized by (minimal) symplectic 4-manifolds or by Lefschetz fibrations. In particular, it is expected that for any symplectic 4-manifold $X$ not diffeomorphic to the blow-up of a ruled surface we have $\chi(X) \geq 0$ ([10, page 579] and [25. Conjecture 2.10]), and for a Lefschetz fibration over the sphere we have $\sigma \leq 0-$ cf. [16, Problems 7.4 and 7.5].

Even though there is significant progress in signature computations [8, 21, 22, and the signature of a Lefschetz fibration is algorithmically computable, its global properties are not known except for hyperelliptic Lefschetz fibrations.

The minimal number of singular fibers of Lefschetz fibrations seems to be closely related to these questions; this problem has been extensively studied in 1, 12, 14, 16, 20, 25. (In what follows, we will always assume that the fibration map is injective on the set of critical points, hence the number of singular points and the number of singular fibers coincide. To avoid trivial examples, we will also assume that all Lefschetz fibrations are relatively minimal and admit at least one singular fiber.) Suppose that $\Sigma_{h}$ denotes the closed, oriented surface of genus $h$. Let the positive integer $N(g, h)$ be defined as

$N(g, h)=$

$\min \left\{k \mid\right.$ there is a genus- $g$ Lefschetz fibration over $\Sigma_{h}$ with $k>0$ singular fibers $\}$.

Date: April 23, 2022.

2010 Mathematics Subject Classification. 57N13, 53D35.

Key words and phrases. Lefschetz fibration, Mapping class group.

We would like to thank the referee for many valuable comments and F. Catanese for pointing out an error in an earlier version of this paper. AS was partially supported by ERC Advanced Grant LDTBud and by the Momentum program of the Hungarian Academy of Sciences. KY was partially supported by the Basic Science Research Program through the National Research Foundation of Korea (NRF) funded by the Ministry of Education (2015R1D1A1A01058941) and by Sungshin Women's University study abroad program. 
The value of $N(g, h)$ has already been computed except $N(g, 0)$ for $g \geq 3, N(g, 1)$ for $g \geq 2$ and $N(2,2)$. Recently Baykur and Korkmaz [1 found an interesting relation in the mapping class group $\mathcal{M}_{2}^{1}$ of the genus-2 surface with one boundary component, and by using this relation they showed that $N(2,0)=7$. Furthermore, using the 8-holed torus relation [15] and the Matsumoto-Cadavid-Korkmaz relation [3, 13, 19], Hamada [12 found an upper bound for $N(g, 1)$ : he showed that $N(g, 1) \leq 4$ if $g \geq 5$ and $N(g, 1) \leq 6$ if $g=3,4$.

In this paper we estimate $N(g, 1)$ further by using some constraints on the signature of a Lefschetz fibration over the torus $T^{2}$.

Theorem 1.1. Let $f: X \rightarrow T^{2}$ be a genus-g Lefschetz fibration. Then $f$ has at least three singular fibers, that is, $N(g, 1) \geq 3$ for $g \geq 1$.

By lifting the Baykur-Korkmaz relation to $\mathcal{M}_{2}^{2}$ we also show

Theorem 1.2. If $g \geq 3$, then there is a genus-g Lefschetz fibration over the torus with 5 singular fibers, that is, $N(g, 1) \leq 5$ for $g \geq 3$.

When combined these two theorems with sharper results of Hamada 12 and some special properties of the mapping class group of the genus- 2 surface, we get:

Corollary 1.3. For the minimal number $N(g, 1)$ of singular fibers in a genus- $g$ Lefschetz fibration over the torus we have

(1) $3 \leq N(g, 1) \leq 4$ for $g \geq 5$,

(2) $3 \leq N(g, 1) \leq 5$ for $g=3,4$,

(3) $N(2,1)=7$ and $N(1,1)=12$.

Remark 1.4. In [5], the existence of a complex surface $S$ with $b_{1}(S)=2, \sigma(S)=1$ and $\chi(S)=3$ is shown. The Albanese map $S \rightarrow T^{2}$ is studied in [4, where it has been shown that this map can be perturbed to a symplectic Lefschetz fibration of genus 19 with exactly 3 singular fibers. When combined this construction with the result above, we get $N(19,1)=3$. The exact value of $N(g, 1)$ for other values of $g$ is still open.

\section{A LOWER BOUND ON $N(g, 1)$}

We start our discussion by recalling some relevant results. (For basic notions and constructions of Lefschetz fibrations see [11, Chapter 8]. Unless otherwise stated, in the following we will consider only relatively minimal Lefschetz fibrations with at least one singular fiber, and will assume that the fibration map is injective on the set of critical points.)

Theorem 2.1 ([17, 23, 24]). Let $X$ be a connected smooth closed oriented 4manifold and $f: X \rightarrow B$ be a Lefschetz fibration with fiber $F$. If $g(F) \geq 2$ and $g(B) \geq 1$, then

$$
2(g(F)-1)(g(B)-1) \leq c_{1}^{2}(X) \leq 5 c_{2}(X) .
$$

In particular, a Lefschetz fibration over the torus with fiber genus at least 2 satisfies $0 \leq c_{1}^{2}(X) \leq 5 c_{2}(X)$.

Theorem 2.2 (2, 7]). Let $X$ be a connected smooth closed oriented 4-manifold and $f: X \rightarrow B$ be a Lefschetz fibration with fiber $F$ where $g(F) \geq 1$ and $g(B) \geq 1$. 
Let $n$ be the number of nonseparating and $s$ be the number of separating vanishing cycles. Then,

$$
s \leq 6(3 g(F)-1)(g(B)-1)+5 n .
$$

In particular, for a Lefschetz fibration over the torus we have $s \leq 5 n$.

The following proposition will be used to show that $N(g, 1) \neq 2$.

Proposition 2.3. Let $f: X \rightarrow T^{2}$ be a genus-g Lefschetz fibration over the torus $T^{2}$ which has $k$ singular fibers, $n$ of them with nonseparating vanishing cycles and $s$ of them with separating vanishing cycles. Then

$$
-\frac{2}{3} k \leq \sigma(X) \leq n-s-1
$$

Proof. Note first that for a Lefschetz fibration $f: X \rightarrow B$ of fiber genus $g$ and base genus $h$ and with $k$ singular fibers we have $\chi(X)=4(g-1)(h-1)+k$; in particular if $B$ is diffeomorphic to the torus, then $\chi(X)=k$.

The first inequality of the proposition now follows from Equation (2.1), as

$$
0 \leq c_{1}^{2}(X)=3 \sigma(X)+2 \chi(X)=3 \sigma(X)+2 k .
$$

For the second inequality, let us consider an $m$-fold (unbranched) covering

$$
\phi_{m}: T^{2} \rightarrow T^{2}
$$

of the base torus $T^{2}$ and pull back the Lefschetz fibration $f: X \rightarrow T^{2}$ along this map:

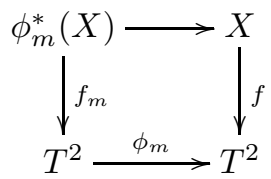

The Euler characteristic, the signature and the number of singular fibers all multiply by $m$, hence we get:

(1) $\chi\left(\phi_{m}^{*}(X)\right)=m k=m n+m s$

(2) $b_{1}\left(\phi_{m}^{*}(X)\right) \leq 2 g+2$

(3) $\chi\left(\phi_{m}^{*}(X)\right)=2-2 b_{1}\left(\phi_{m}^{*}(X)\right)+b_{2}\left(\phi_{m}^{*}(X)\right)=m \chi(X)=m k$

(4) $\sigma\left(\phi_{m}^{*}(X)\right)=m \sigma(X)$.

Therefore

$$
\begin{aligned}
& \sigma\left(\phi_{m}^{*}(X)\right)=b_{2}^{+}\left(\phi_{m}^{*}(X)\right)-b_{2}^{-}\left(\phi_{m}^{*}(X)\right)=b_{2}\left(\phi_{m}^{*}(X)\right)-2 b_{2}^{-}\left(\phi_{m}^{*}(X)\right)= \\
& =\chi\left(\phi_{m}^{*}(X)\right)-2+2 b_{1}\left(\phi_{m}^{*}(X)\right)-2 b_{2}^{-}\left(\phi_{m}^{*}(X)\right) \leq m k+4 g+2-2 b_{2}^{-}\left(\phi_{m}^{*}(X)\right),
\end{aligned}
$$

and we will estimate $b_{2}^{-}\left(\phi_{m}^{*}(X)\right)$.

In the mapping class group of the fiber, let us denote $y x y^{-1}$ by $[x]^{y}$. We will consider $a_{i}, b_{i}$ as a right handed Dehn twists along corresponding simple closed curves on the fiber $F$. In the following we use the function notation for a product in the mapping class group, i.e., $a b=a \circ b$ means that $b$ is applied first and then we apply $a$. For simplicity we will use the same letter for a Dehn twist and its corresponding isotopy class of simple closed curve.

Since the 4-manifold $X$ admits a Lefschetz fibration over $T^{2}$, it is characterized by the equivalence class of the monodromy factorization of the fibration of the form:

$$
a_{1} a_{2} \cdots a_{s} b_{1} b_{2} \cdots b_{n}=[\gamma, \delta]
$$


for some right handed Dehn twists $a_{i}(i=1, \cdots, s)$ along separating vanishing cycles and right handed Dehn twists $b_{j}(j=1,2, \cdots, n)$ along nonseparating vanishing cycles, and some elements $\gamma, \delta$ in the mapping class group of the fiber $F$.

The monodromy factorization of the Lefschetz fibration $\phi_{m}^{*}(X)$ has the form

$$
\tilde{a}_{1} \tilde{a}_{2} \cdots \tilde{a}_{m s} \tilde{b}_{1} \tilde{b}_{2} \cdots \tilde{b}_{m n}=\left[\gamma_{m}, \delta_{m}\right]
$$

for some right handed Dehn twists $\tilde{a}_{i}(i=1, \cdots, m s)$ along separating vanishing cycles and right handed Dehn twists $\tilde{b}_{j}(j=1, \cdots, m n)$ along nonseparating vanishing cycles and some elements $\gamma_{m}, \delta_{m}$ in the mapping class group of the fiber F.

Each separating vanishing cycle gives rise to an embedded surface (hence a second homology element $A_{i}$ ) of self-intersection $(-1)$ which is part of the corresponding singular fiber. The Lefschetz fibration $\phi_{m}^{*}(X)$ therefore has $m s$ many such disjoint surfaces

$$
\left\{A_{1}, A_{2}, \cdots, A_{m s}\right\} \text {. }
$$

Now we claim that there is a surface of negative self intersection in a neighbourhood of singular fibers $\prod_{i=1}^{2 g+1} \tilde{b}_{(2 g+1) j+i}$ for each $j=0, \cdots,\left[\frac{m n}{2 g+1}\right]-1$.

Since $\operatorname{dim} H_{1}(F ; \mathbb{Z})=2 g$,

$$
\left\{\tilde{b}_{(2 g+1) j+1}, \tilde{b}_{(2 g+1) j+2}, \cdots, \tilde{b}_{(2 g+1)(j+1)}\right\}
$$

is linearly dependent for each $j=0, \cdots,\left[\frac{m n}{2 g+1}\right]-1$. For each $j$ all argument will be the same, hence to keep the notation simple, we assume $j=0$. Obviously we can find a positive integer $\ell \leq 2 g+1$ such that

$$
\left\{\tilde{b}_{1}, \tilde{b}_{2}, \cdots, \tilde{b}_{\ell-1}\right\}
$$

is linearly independent and

$$
\left\{\tilde{b}_{1}, \tilde{b}_{2}, \cdots, \tilde{b}_{\ell}\right\}
$$

is linearly dependent. Then we can find some integers $n_{v}$ such that

$$
\sum_{v=1}^{\ell-1} n_{v} \tilde{b}_{v}+n_{\ell} \tilde{b}_{\ell}=0, n_{\ell} \neq 0
$$

in $H_{1}(F ; \mathbb{Z})$. Since this implies the same identity in $H_{1}\left(\partial\left(F \times D^{2}\right) ; \mathbb{Z}\right)$, there is an element $\alpha \in H_{2}\left(F \times D^{2}, \partial\left(F \times D^{2}\right) ; \mathbb{Z}\right)$ which can be represented by a surface $S_{\alpha}$ in $F \times D^{2}$ with $\sum_{v=1}^{\ell}\left|n_{v}\right|$ boundary components, $\left|n_{v}\right|$ copies of $\tilde{b}_{v}$ for each $v=1, \cdots, \ell$, all located in different fibers. Using the Lefschetz thimbles of the singular fibers in $X$ corresponding to $\tilde{b}_{1}, \tilde{b}_{2}, \cdots, \tilde{b}_{\ell}$ we get a closed, oriented surface $S$ in $X$.

Next we show that the self-intersection of $S$ is negative. Decompose $S$ as $S_{\alpha}$ together with the $\sum_{v=1}^{\ell}\left|n_{v}\right|$ Lefschetz thimbles. When framing the $\sum_{v=1}^{\ell}\left|n_{v}\right|$ boundary circles of $S_{\alpha}$ with the framing these circles get from the fiber of the fibration, the self-intersection $[S]^{2}$ can be computed as the sum of $\left[S_{\alpha}\right]^{2}$ (with respect to the above framing at the boundary) together with the self-intersections of the thimbles (again, with the same framings at their boundaries). The relative self-intersection of the Lefschetz thimble is equal to $(-1)$, hence the contribution of the $\sum_{v=1}^{\ell}\left|n_{v}\right|$ thimbles in our case is $-\sum_{v=1}^{\ell} n_{v}^{2}$ (since $\tilde{b}_{v}$ appears $\left|n_{v}\right|$ times, hence this thimble is used $\left|n_{v}\right|$ times).

In computing the self-intersection of the surface-with-boundary $S_{\alpha} \subset F \times D^{2}$ (with the framings along the $\sum_{v=1}^{\ell}\left|n_{v}\right|$ boundary components as fixed above) we 
argue as follows. We attach $\ell$ two-handles to $F \times D^{2}$ along the simple closed curves $\tilde{b}_{1}, \tilde{b}_{2}, \cdots, \tilde{b}_{\ell}$, with coefficient 0 (measured with respect to the framing given above). The resulting 4-manifold $\bar{X}$ satisfies $H_{2}(\bar{X} ; \mathbb{Z})=\mathbb{Z} \oplus \mathbb{Z}$ : the first $(\ell-1)$ handles are attached along homologically nontrivial and linearly independent curves, hence reduce $H_{1}$ and do not change $H_{2}$, while the last 2-handle is attached along a curve homologically depending on the first $(\ell-1)$ curves, hence this last attachment increases the rank of $H_{2}$ by one. The two generators of $H_{2}(\bar{X} ; \mathbb{Z})$ can be given by $F \times\{0\}$ and the capped-off surface we get from $S_{\alpha}$, and which we will denote by $\Sigma$. These classes can be represented by disjoint surfaces, since $S_{\alpha}$ can be pushed into $\partial\left(F \times D^{2}\right)$; consequently $[F \times\{0\}] \cdot[\Sigma]=0$. The self-intersection of the homology class represented by $F \times\{0\}$ is also zero, hence the sign of the self-intersection of $\Sigma$ is equal to the sign of the signature of $\bar{X}$. The signature $\sigma(\bar{X})$, however, is easy to compute: the first $(\ell-1)$ attachments do not change $\sigma\left(F \times D^{2}\right)=0$ (since they do not change $H_{2}\left(F \times D^{2} ; \mathbb{Z}\right)$ either), and by Wall's nonadditivity of signature ([21, Theorem 3]) the last handle attachment also leaves $\sigma$ unchanged, since $\left|n_{\ell}\right|$ copies of the simple closed curve corresponding to the 0 -framing in the 2-handle attachment along $\tilde{b}_{\ell}$ bounds a surface (cf. the formula in [21]).) This shows that the self-intersection of $\Sigma$ is zero in $\bar{X}$, hence the self-intersection of $S_{\alpha}$ in $F \times D^{2}$ (with the framings along the boundary components as discussed above) is 0 , implying that $S \subset X$ is a surface of self-intersection $-\sum_{v=1}^{\ell} n_{v}^{2}$.

The surface $S$ is obviously disjoint from the $A_{i}$ 's (coming from separating vanishing cycles), and the $S$ 's for different $j$ 's are also disjoint. Therefore we get

implying

$$
b_{2}^{-}\left(\phi_{m}^{*}(X)\right) \geq m s+\left[\frac{m n}{2 g+1}\right],
$$

$$
m \sigma(X) \leq m k-2+2(2 g+2)-2\left(m s+\left[\frac{m n}{2 g+1}\right]\right)
$$

for each positive integer $m$. This implies

$$
\sigma(X) \leq k-2 s-\frac{2 n}{2 g+1},
$$

and since $\sigma(X)$ is an integer, together with the fact that $n>0$ (Theorem 2.2) we get

$$
\sigma(X) \leq k-2 s-1 .
$$

Proof of Theorem 1.1. Suppose that there is a genus- $g$ Lefschetz fibration $f: X \rightarrow$ $T^{2}$ with $k=n+s$ singular fibers. Then, the following conditions have to be satisfied:

(2.4) $s \leq 5 n$ (Theorem 2.2).

It is known that $N(1,1)=12[18, N(g, 1)>1$ for $g \geq 1$ [14, and $N(2,1) \geq 6$ because (2.4) implies $n>0$ [19, 20]. Notice that $N(g, 1)>1$ also follows from (2.2): if $k=1$ then (2.2) implies $-\frac{2}{3} \leq \sigma(X) \leq 0$, so $\sigma(X)=0$, contradicting (2.3).

For $k=2$, we also use (2.3): there is no integer $\sigma(X)$ satisfying

$$
-\frac{4}{3} \leq \sigma(X) \leq 2-2 s-1=1-2 s
$$


and $4 \mid(\sigma(X)+2)$.

Remark 2.4. If there is a Lefschetz fibration $X$ over $T^{2}$ with $k=3$, then by (2.2) and (2.3) we have $\sigma(X)=1, \chi(X)=3$ and $(n, s)=(3,0)$. As already pointed out in Remark 1.4, such a fibration has been claimed in 4 for fiber genus 19, based on the construction of a complex surface $S$ in $[\underline{5}$.

\section{UPPER BOUNDS ON $N(g, 1)$}

Hamada proved the following upper bounds for $N(g, 1), g \geq 3$.

Theorem $3.1([12])$. For the minimal number $N(g, 1)$ of singular fibers in a genus$g$ Lefschetz fibration over the torus we have

(1) $N(g, 1) \leq 6$ for $g \geq 3$,

(2) $N(g, 1) \leq 4$ for $g \geq 5$.

The proof of this result uses the Matsumoto-Cadavid-Korkmaz word [3, 13, 19, implying $N(g, 1) \leq 6$ for $g \geq 3$, and the 8-holed torus relation (originally obtained by Korkmaz and Ozbagci [15]), implying $N(g, 1) \leq 4$ for $g \geq 5$.

By modifying the 4-chain relation, Baykur and Korkmaz [1 found an interesting relation in $\mathcal{M}_{2}^{1}$. In the following we lift the Baykur-Korkmaz relation (a relation which is obtained from Equation (3.1) below by capping off the boundary circle $\delta_{0}$ with a disk) to $\mathcal{M}_{2}^{2}$, and using this lift we improve the upper bounds for $N(3,1)$ and $N(4,1)$. This result was predicted by Hamada and a sketch is given in [12].

Proposition 3.2. There is a relation

$$
x_{1} x_{2} x_{3} x_{4} y_{1} y_{2} y_{3}=\delta_{0} \delta_{2}
$$

in the mapping class group $\mathcal{M}_{2}^{2}$ of the genus-2 surface with two boundary components $\delta_{0}$ and $\delta_{2}$, where $x_{i}$ are nonseparating vanishing cycles and $y_{i}$ are separating vanishing cycles.

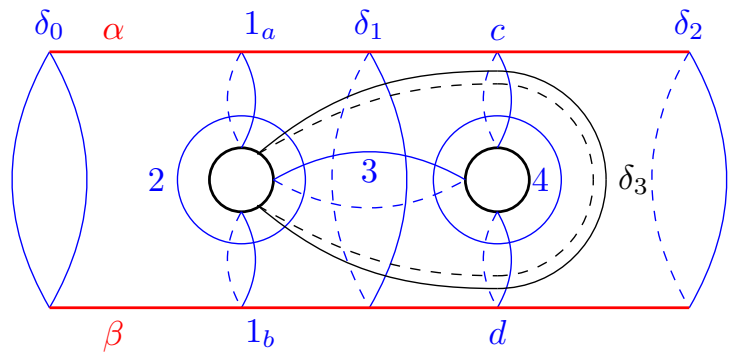

FiguRE 1. Curves for the chain relation

Proof. By using a sequence of braid relations, we get

$$
\begin{aligned}
& k 1_{a} 234=1_{a} 234 \ell, \quad k 1_{b} 234=1_{b} 234 \ell, \quad 21_{a} 234=1_{a} 2341_{a}, \quad 21_{b} 234=1_{b} 2341_{b}, \\
& \ell 4321_{a}=4321_{a} k, \quad \ell 4321_{b}=4321_{b} k, \quad 1_{a} 4321_{a}=4321_{a} 2, \quad 1_{b} 4321_{b}=4321_{b} 2,
\end{aligned}
$$


where $k \in\{3,4\}$ and $k-\ell=1$. This implies that the elements of the set $\left\{\left(1_{a} 1_{b}\right),\left(21_{a} 1_{b} 2\right),\left(321_{a} 1_{b} 23\right),\left(4321_{a} 1_{b} 234\right)\right\}$ commute with each other and

$$
\begin{aligned}
\left(1_{a} 2341_{b} 234\right)^{2}\left(1_{a} 234\right) & =1_{a} 231_{b} 231_{a} 231_{b} 234321_{b} 1_{a} 234 \\
& =1_{a} 21_{b} 21_{a} 2321_{a} 1_{b} 234321_{b} 1_{a} 234 \\
& =\left(1_{a} 1_{b}\right)\left(21_{a} 1_{b} 2\right)\left(321_{a} 1_{b} 23\right)\left(4321_{a} 1_{b} 234\right), \\
\left(1_{b} 234\right)\left(1_{a} 2341_{b} 234\right)^{2} & =\left(1_{a} 1_{b}\right)\left(21_{a} 1_{b} 2\right)\left(321_{a} 1_{b} 23\right)\left(4321_{a} 1_{b} 234\right) .
\end{aligned}
$$

Therefore

$$
\left(1_{a} 2341_{b} 234\right)^{5}=\left(1_{a} 1_{b}\right)^{2}\left(21_{a} 1_{b} 2\right)^{2}\left(321_{a} 1_{b} 23\right)^{2}\left(4321_{a} 1_{b} 234\right)^{2} .
$$

First we prove the following relation in $\mathcal{M}_{2}^{2}$ :

$$
\left(1_{a} 2341_{b} 234\right)^{5}=\delta_{0}^{3} \delta_{2}
$$

(Note that if we attach a disk along $\delta_{0}$, then the above relation reduces to the usual 4-chain relation $(1234)^{10}=\delta_{2}$, hence we can regard it as a lift of the 4-chain relation to $\mathcal{M}_{2}^{2}$.) The 3 -chain relation implies

$$
\left(1_{a} 1_{b}\right)^{2}\left(21_{a} 1_{b} 2\right)^{2}=\left(\left(1_{a} 1_{b}\right)\left(21_{a} 1_{b} 2\right)\right)^{2}=\left(1_{a} 21_{b}\right)^{4}=\delta_{0} \delta_{1} .
$$

Since the genus- 2 surface $\Sigma_{2}^{2}$ with two boundary components can be decomposed into eight hexagons by cutting it along seven simple closed curves $1_{a}, 1_{b}, 2,3,4, c$, $d$ and two $\operatorname{arcs} \alpha, \beta$ as in Figure 1, we will prove

$$
\left(321_{a} 1_{b} 23\right)^{2}\left(4321_{a} 1_{b} 234\right)^{2}=\delta_{0}^{2} \delta_{1}^{-1} \delta_{2}
$$

by showing that nine circles $1_{a}, 1_{b}, 2,3,4, c, d, \delta_{0}, \delta_{2}$ and two $\operatorname{arcs} \alpha, \beta$ are fixed (up to isotopy) under the map

$$
\left(\delta_{0}^{2} \delta_{1}^{-1} \delta_{2}\right)^{-1}\left(321_{a} 1_{b} 23\right)^{2}\left(4321_{a} 1_{b} 234\right)^{2} .
$$

- $\delta_{0}$ and $\delta_{2}$ are clearly fixed under the map (3.5).

- $c$ and $d$ are fixed under $\left(4321_{a} 1_{b} 234\right)^{2}$ and $\left(321_{a} 1_{b} 23\right)$, hence are fixed under the map (3.5).

- $\left(321_{a} 1_{b} 23\right)\left(4321_{a} 1_{b} 234\right)$ maps $1_{a}, 1_{b}$ and 2 to themselves with the same orientation. So $1_{a}, 1_{b}$ and 2 are fixed under the map (3.5).

- $\left(321_{a} 1_{b} 23\right)\left(4321_{a} 1_{b} 234\right)$ maps 4 to itself but with opposite orientation. So 4 is fixed under the map $\left(321_{a} 1_{b} 23\right)^{2}\left(4321_{a} 1_{b} 234\right)^{2}$ and therefore by (3.5).

- $\left(4321_{a} 1_{b} 234\right)$ maps the simple closed curve 3 to itself but with opposite orientation. The image of the simple closed curve 3 under the map $\left(321_{b} 1_{a} 23\right)^{2}$ is $\delta_{1}^{-1}(3)$. Therefore 3 is fixed under the map (3.5).

- $\left(321_{a} 1_{b} 23\right)^{2}\left(4321_{a} 1_{b} 234\right)^{2}$ maps $\alpha$ to $\delta_{0}^{2} \delta_{1}^{-1} \delta_{2}(\alpha)$ and $\beta$ to $\delta_{0}^{2} \delta_{1}^{-1} \delta_{2}(\beta)$. So $\alpha$ and $\beta$ are fixed under the map (3.5).

Equations (3.3) and (3.4) then imply Equation (3.2).

Now we will check that there is a lift of the Baykur-Korkmaz word to $\mathcal{M}_{2}^{2}$. By following the steps of the reverse engineering method applied by Baykur and Korkmaz in [1], we get

$$
\begin{aligned}
& \left(321_{a} 1_{b} 23\right)^{2}\left(4321_{a} 1_{b} 234\right)^{2}=\left(321_{a} 1_{b} 23\right)\left(4321_{a} 1_{b} 234\right)^{2}\left(321_{a} 1_{b} 23\right)= \\
& =3421_{a} 1_{b} 3231_{a} 1_{b} 2(34)^{3} 21_{a} 1_{b} 2321_{a} 1_{b} 243 .
\end{aligned}
$$

This implies

$$
\delta_{0}^{3} \delta_{2}=\delta_{0} \delta_{1} 3421_{a} 1_{b} 3231_{a} 1_{b} 2(34)^{3} 21_{a} 1_{b} 2321_{a} 1_{b} 243,
$$


and since 43 commutes with $\delta_{0}^{3} \delta_{2}$, we can perform a cyclic permutation; a sequence of Hurwitz moves then gives

$$
\begin{aligned}
\delta_{0}^{3} \delta_{2} & =\delta_{0}\left[\delta_{1}\right]^{43} 433421_{a} 1_{b} 3231_{a} 1_{b} 2(34)^{3} 21_{a} 1_{b} 2321_{a} 1_{b} 2 \\
& =\delta_{0}\left[\delta_{1}\right]^{43}[2]^{4334}[2]^{43341_{a} 1_{b} 3}[2]^{43341_{a} 1_{b} 331_{a} 1_{b}}\left(1_{a} 1_{b}\right)^{2}\left(21_{a} 1_{b} 2\right)^{2}(34)^{6}[3]^{\left(21_{a} 1_{b} 2\right)^{-1}} \\
& =\delta_{0}\left[\delta_{1}\right]^{43}[2]^{4334}[2]^{43341_{a} 1_{b} 3}[2]^{43341_{a} 1_{b} 331_{a} 1_{b}} \delta_{0} \delta_{1} \delta_{3}[3]^{\left(21_{a} 1_{b} 2\right)^{-1}}
\end{aligned}
$$

where $\delta_{3}$ is the right handed Dehn twist along the curve $\delta_{3}$ of Figure 1, Therefore

$$
\delta_{0} \delta_{2}=[3]^{\left(21_{a} 1_{b} 2\right)^{-1}}\left[\delta_{1}\right]^{43}[2]^{4334}[2]^{43341_{a} 1_{b} 3}[2]^{43341_{a} 1_{b} 331_{a} 1_{b}} \delta_{1} \delta_{3} .
$$

By choosing

$$
\begin{aligned}
& x_{1}=[3]^{\left(21_{a} 1_{b} 2\right)^{-1}}, \quad x_{2}=[2]^{4334}, \quad x_{3}=[2]^{43341_{a} 1_{b} 3}, \quad x_{4}=[2]^{43341_{a} 1_{b} 331_{a} 1_{b}}, \\
& y_{1}=\left[\delta_{1}\right]^{\left(x_{2} x_{3} x_{4}\right)^{-1} 43}, \quad y_{2}=\delta_{1}, \quad y_{3}=\delta_{3},
\end{aligned}
$$

the conclusion follows.

Remark 3.3. Notice that Relation (3.2) shows the existence of a (-3)-section of the Lefschetz fibration determined by the left-hand-side of the relation. This fibration was already discussed by Fuller [9, page 164].

Proof of Theorem 1.2. Let us cap off the two boundary components $\delta_{0}, \delta_{2}$ of $\Sigma_{2}^{2}$ by a cylinder so that we get a closed surface $\Sigma_{3}$ of genus 3 . Then each vanishing cycle except $y_{3}$ is nonseparating and $\Sigma_{3}-\left\{\delta_{0}, x_{2}\right\}$ and $\Sigma_{3}-\left\{\delta_{2}, x_{1}\right\}$ are connected, $\delta_{0}$ is disjoint from $x_{2}$, and $\delta_{2}$ is disjoint from $x_{1}$. As it is explained in [6, 14, there is a map $\psi: \Sigma_{3} \rightarrow \Sigma_{3}$ satisfying

$$
\psi\left(\delta_{0}\right)=x_{1}, \quad \psi\left(x_{2}\right)=\delta_{2},
$$

implying that

$$
x_{3} x_{4} y_{1} y_{2} y_{3}=\left[\delta_{0} x_{2}^{-1}, \psi\right] .
$$

So there is a genus-3 Lefschetz fibration over $T^{2}$ with 5 singular fibers, 4 of them are nonseparating and 1 of them is separating.

We get the same result for $g \geq 4$, since we can cap off the two boundary components $\delta_{0}$ and $\delta_{2}$ by a twice punctured genus- $(g-3)$ surface.

As an application, we get

Proof of Corollary 1.3. Theorems 1.1, 3.1 and 1.2 immediately imply (1) and (2).

For (3), it is already known that $N(1,1)=12$ and $6 \leq N(2,1) \leq 7$. So we only need to show that there is no genus-2 Lefschetz fibration $X$ over $T^{2}$ with 6 singular fibers. It is well known that $\mathcal{M}_{2}$ is the hyperelliptic mapping class group, consequently $n+2 s \equiv 0(\bmod 10)$. Therefore the only possible case for $k=6$ is $(n, s)=(2,4)$. But Proposition 2.3 excludes this case because there is no $\sigma(X) \in \mathbb{Z}$ such that

$$
-\frac{12}{3} \leq \sigma(X) \leq n-s-1=2-4-1=-3,
$$

and $4 \mid(6+\sigma(X))$. 


\section{REFERENCES}

1. I. Baykur and M. Korkmaz, Small Lefschetz fibrations and exotic 4-manifolds, arXiv:1510.00089 math.GT]

2. V. Braungardt and D. Kotschick, Clustering of critical points in Lefschetz fibrations and the symplectic Szpiro inequality, Trans. Amer. Math. Soc. 355 (2003), no. 8, 3217-3226. MR 1974683

3. C. Cadavid, On a remarkable set of words in the mapping class group, ProQuest LLC, Ann Arbor, MI, 1998, Thesis (Ph.D.)-The University of Texas at Austin. MR 2699379

4. D. Cartwright, V. Koziarz, and S.-K. Yeung, On the Cartwright-Steger surface, arXiv:1412.4137 math.AG]

5. D. Cartwright and T. Steger, Enumeration of the 50 fake projective planes, C. R. Math. Acad. Sci. Paris 348 (2010), no. 1-2, 11-13. MR 2586735

6. H. Endo, M. Korkmaz, D. Kotschick, B. Ozbagci, and A. Stipsicz, Commutators, Lefschetz fibrations and the signatures of surface bundles, Topology 41 (2002), no. 5, 961-977. MR 1923994

7. H. Endo and D. Kotschick, Bounded cohomology and non-uniform perfection of mapping class groups, Invent. Math. 144 (2001), no. 1, 169-175. MR 1821147

8. H. Endo, Meyer's signature cocycle and hyperelliptic fibrations, Math. Ann. 316 (2000), no. 2, 237-257. MR 1741270

9. T. Fuller, Diffeomorphism types of genus 2 Lefschetz fibrations, Math. Ann. 311 (1998), no. 1, 163-176. MR 1624287

10. R. Gompf, A new construction of symplectic manifolds, Ann. of Math. (2) 142 (1995), no. 3, 527-595. MR 1356781

11. R. Gompf and A. Stipsicz, 4-manifolds and Kirby calculus, Graduate Studies in Mathematics, 20. American Mathematical Society, Providence, RI, 1999.

12. N. Hamada, Upper bounds for the minimal number of singular fibers in a Lefschetz fibration over the torus, Michigan Math. J. 63 (2014), no. 2, 275-291. MR 3215551

13. M. Korkmaz, Noncomplex smooth 4-manifolds with Lefschetz fibrations, Internat. Math. Res. Notices (2001), no. 3, 115-128. MR 1810689

14. M. Korkmaz and B. Ozbagci, Minimal number of singular fibers in a Lefschetz fibration, Proc. Amer. Math. Soc. 129 (2001), no. 5, 1545-1549. MR 1713513

15. M. Korkmaz and B. Ozbagci, On sections of elliptic fibrations, Michigan Math. J. 56 (2008), no. $1,77-87$. MR 2433657

16. M. Korkmaz and A. Stipsicz, Lefschetz fibrations on 4-manifolds, Handbook of Teichmüller theory. Vol. II, IRMA Lect. Math. Theor. Phys., vol. 13, Eur. Math. Soc., Zürich, 2009, pp. 271-296. MR 2497784

17. T.-J. Li, Symplectic Parshin-Arakelov inequality, Internat. Math. Res. Notices (2000), no. 18, 941-954. MR 1792283

18. Y. Matsumoto, Diffeomorphism types of elliptic surfaces, Topology 25 (1986), no. 4, 549-563. MR 862439

19. Y. Matsumoto, Lefschetz fibrations of genus two-a topological approach, Topology and Teichmüller spaces (Katinkulta, 1995), World Sci. Publ., River Edge, NJ, 1996, pp. 123-148. MR 1659687

20. N. Monden, On minimal number of singular fibers in a genus-2 Lefschetz fibration, Tokyo J. Math. 35 (2012), no. 2, 483-490. MR 3058720

21. B. Ozbagci, Signatures of Lefschetz fibrations, Pacific J. Math. 202 (2002), no. 1, 99-118. MR 1883972

22. I. Smith, Lefschetz fibrations and the Hodge bundle, Geom. Topol. 3 (1999), 211-233 MR 1701812

23. A. Stipsicz, Chern numbers of certain Lefschetz fibrations, Proc. Amer. Math. Soc. 128 (2000), no. 6, 1845-1851. MR 1641113

24. A. Stipsicz, Erratum to: "Chern numbers of certain Lefschetz fibrations" [Proc. Amer. Math. Soc. 128 (2000), no. 6, 1845-1851; MR1641113 (2000j:57062)], Proc. Amer. Math. Soc. 128 (2000), no. 9, 2833-2834 (electronic). MR 1766607

25. A. Stipsicz, Singular fibers in Lefschetz fibrations on manifolds with $b_{2}^{+}=1$, Topology Appl. 117 (2002), no. 1, 9-21. MR 1874001 
MTA, Rényi institute of Mathematics, Budapest, Hungary

E-mail address: stipsicz.andras@renyi.mta.hu

Department of mathematics, Sungshin Women's University, Seoul 02844, Korea

E-mail address: kyun@sungshin.ac.kr 